Instability, Chaos and Predictability in Celestial Mechanics and Stellar Dynamics
Copyright 1992 by Nova Science Publishers, Inc. All rights of reproduction in any form reserved.

\title{
ChaOtic STEllar DYNamics DURING COLLECTIVE INTERACTIONS
}

\author{
D. Subbarao \\ Fusion Studies Program(Centre of Energy Studies) \\ R. Uma \\ Department of Physics, Indian Institute of Technology, Delhi-110016, India
}

\section{ABSTRACT .}

We prove a basic result for collisionless galactic models that collective motion not only introduces Landau damping but also intrinsic chaos of typical star dynamics in the phase plane when a small perturbative wave disturbance is present. The Melnikov method is used; the consequences of the chaos and quasilinear diffusion are pointed out.

\section{INTRODUCTION}

The inverse square gravitational law is responsible for long range interactions in gravitationally interacting mass bodies and has many interesting consequences; gentle relaxation and collective interaction are two of them. The understanding of wave propagation in a collection of stars is based on the collective interaction theory; an interesting implication is the collisionless or Landau damping of waves whenever they can exist [1-4]. Careful examination of such collisionless phenomena could be very rewarding.

In this paper we reexamine the problem of collective interaction in the light of the fact that a collection of stellar objects could give rise to chaotic motion as well as regular motion just as in any other dynamical system [5],[6]. Collective interaction can be associated with a continuous spectrum in phase space [7],[9] through the so-called Van Kampen Case Siwert modal structure $[7],[9],[11]$. Because of the recent discovery of its relation to intrinsic chaos [10], [11] we find it important to point out that whenever wave structure is allowed in a gravitationally interacting stellar medium, the dynamics of an individual stellar object in phase space should be expected to be chaotic; the chaos itself 
immediately manifests in the form of quasilinear diffusion $[2],[4]$.

Only the simplest physical model is considered here since the aim is to put on record the basic phenomenon to be expected; more detailed specific models are left out for future studies. Hence, a homogeneous distribution of stellar objects is considered and spatial finiteness effects of the distribution of such objects are not dealt with; the model considered is as in Chapters 15 \& 16 of Ref. 3 . The regime of waves of shorter wavelengths than Jeans length is of interest; the alternate regime gives $r$ ise to the collapse of the collection of stellar objects not giving rise to chaotic dynamics (see last part of this paper). The treatment follows that of a similar proof in plasma physics [11] which resulted because of application to thermo nuclear fusion.

The starting point for examining individual star behaviour in a collection of similar objects would have been the collisionless Boltzman (Vlasov) equation as is usual in stellar dynamics [1-4]. However, since we are interested in the dynamics of a typical stellar object and also since the Lie operator $\Lambda_{F}$ is enough to determine all required variables in the problem at a go, we analyse the dynamics in terms of the Dewar equation. The Dewar equation relates the non-autonomous hamiltonian $(\mathrm{H})$ and the canonically transformed hamiltonian $(\bar{H})$ through the generating function $F$ of the canonical transformation.

$$
\frac{\partial \bar{F}}{\partial \mathbf{t}}=\frac{\partial \bar{H}}{\partial g}-L_{F} \bar{H}-\Lambda_{F}^{-1} \frac{\partial H}{\partial g}
$$

where $g$ is perturbation parameter measuring the field or density variation strengths, $L_{F}=i[F$,$] , is the Poisson brac-$ ket operator and the Lie operator is given by,

$$
\Lambda_{F}(g)=\exp \left(i g^{g} L_{F}\left(g^{\prime}\right) d g^{\prime}\right)
$$

For small perturbations, an expansion in the perturbation parameter is carried out in a Deprit series [5] and eq. (1) splits into an infinite hierarchy of coupled equations separated to different orders of $g$. Our interest here is in the 1 inearised form of $\mathrm{Eq}$. (1) and the eigenfunction determination of the resultant equation. This is equivalent to the determination of the eigenspectrum of the linearised Liouville (Valsov) equation for a test star (of mass $m$ ) in the homogeneous collection in the galaxy. The proof of chaos using Melinkov criterion later is in the quasilinear limit. The first order perturbation in the original hamiltonian 
$\mathrm{H}_{\mathrm{O}}\left(=\mathrm{p}^{2} / 2 \mathrm{~m}\right)$ is $\phi \mathrm{m}$ where $\phi$ is the gravitational potential determined from Poisson equation.

$$
\nabla^{2} \phi \quad=-4 \pi \mathrm{Gm} \quad \underset{\mathrm{V}}{f} \mathbf{f} \mathrm{d}^{3} \mathrm{~V}
$$

One can write down an integro-differential equation for $F_{1}$. After integrating out in the momentum space in the perpendicular direction, $p_{1}\left(p=\left(p_{1}, p\right)\right)$ and Fourier transformation in space ( $\sim \exp (i \mathrm{k} . r)$ the longitudinal wave motion is determined by the following transport equation [7],[ 8]

$$
\frac{\partial F_{1}}{\partial t}+i \frac{k}{m} p F_{1}=f n(k, p) k F_{1} d p .
$$

This equation being non self-adjoint there is also an associated adjoint equation

$$
\begin{aligned}
& \frac{\partial \bar{F}_{1}}{\partial \mathrm{t}}+i \frac{k}{\mathrm{~m}} \mathrm{p} \overline{\mathrm{F}}_{1}=n(\mathrm{k}, \mathrm{p}) \mathrm{k} \rho \overline{\mathrm{F}}_{1} \mathrm{dp} ; \\
& n(\mathrm{k}, \mathrm{p})=\frac{4 \pi \mathrm{Gm}^{3}}{\mathrm{k}^{2}} \frac{\partial \mathrm{f}_{\mathrm{o}}}{\partial \mathrm{p}}
\end{aligned}
$$

The structure of the 1 inearised Dewar equations for the generating function $F_{1}$, Eqs. (4) and (5) is very similar to the case of linearised vlasov equation for the perturbed distribution function $f_{1}$ and the two are closely related (see Ref. 11). Indeed, the solutions to the problem can be written down by inspection in terms of the Van Kampen - Case-Siewert solutions [7-9] for the function $f_{1}(x, p, t)$ as done in Ref.11

$$
\begin{aligned}
F(k, p, t)=\sum_{i} a_{i} & \bar{F}_{v i}(k, p) \exp \left(-i k v_{i} t\right) \\
& +\int_{-\infty}^{+\infty} A(v) \bar{F}_{v}(k, p) \exp (-i k v t) d v
\end{aligned}
$$

can be worked out. The descrete part describes periodic orbits while the continuous spectra in the second term describe chaotic motion as outlined briefly below. In a relaxed galaxy with Maxwellian distribution of stars in momentum space, there is no descrete spectrum, it is there whenever there is free energy in the system.

The Melnikov integral using the continuous spectrum in Eq. (6) (for a relaxed galaxy) is of the form: (see for details Ref. 11). 


$$
M\left(t_{0}\right)=g A_{0} \cosh \left(\gamma t_{0}\right) \exp \left(-i \omega t_{0}\right)
$$

where $\gamma$ is Landau damping rate; $\omega$ is the angular frequency of oscillations; $\omega \sim \omega_{G}=\left(4 \pi G \rho_{0}\right)^{1 / 2}$ is the angular frequency of natural collective oscillation of the star collection of uniform mass density $\rho_{o}$ and $A_{o}$ is not $t_{o}$ dependent (see Ref. 11 for details). Eq. (7) essentially states that if $\omega$ is real $M\left(t_{0}\right)$ will pass through zeroes for $M^{\prime}\left(t_{0}\right) \neq 0$ and hence $a$ homoclinic point is indicated. If $\omega$ is pure imaginary there will be then no vanishing out only explosion of $M\left(t_{0}\right)$ and hence there won't be any chaos as indicated by the criterion; the galaxy of stars starts instead a collapse. For complex $\omega$ again there could be chaos.

The above proof of the necessary condition for chaos essentially proves the existence of one homoclinic point at least $\left(M\left(t_{0}\right)=0\right)$ when the stable and unstable manifolds intersect transversely. Because of the Smale-Birkhoff theorem [6] then one deduces the existence of an infinity of such homoclinic points and hence chaos.

The result of the chaotic motion is wave induced quasilinear diffusion which has particularly been discussed in Refs. 1,2 and Ref. 4, Vol. II. In the present case a formal quasilinear diffusion equation:

$$
i \frac{\partial \tilde{f}}{\partial \tilde{t}}=\Phi \tilde{f}
$$

where $\tilde{f}=\Lambda_{F}^{-1} \mathrm{f} ; \Phi=\Lambda_{\mathrm{F}}^{-1} \mathrm{~L}_{\mathrm{H}^{\Lambda} \mathrm{F}}$, can be written down (see references 10 and 11 for details).

Apart from the basic assumptions of the problem already outlined, the chief of them being that realistic finite glaxies have not been considered, the following further $1 \mathrm{im}$ itations should be kept in mind.

Continuous spectrum in configuration space as we11 as in momentum space can exist if a singular intergo-differential equation such as $\mathrm{Eq} .4$ also contains a corresponding singular behaviour in the position coordinate just as it is singular in momentum space. Indeed, such a spatial singularity does occur again in thecontext of an Alfven wave in an inhomogeneous magnetoplasma and has been pointed out in Ref. (13). Quasilinear diffusion because of the resultant chaos in configuration space apart from along momentum axis can be expected to neuteralize the position space inhomogeneity. A similar situation should in general be expected in galactic dynamics where spatial resonances can occur. Such singularities in gas dynamic models of the galaxy could occur at the corotation resonance region or the region of Lindblad resonances. However, dispersive motions of stars and nonlinear 
effects move in fast enough and may smear out the singular behaviour; as such only the transient behavior can show the quasilinear diffusion and chaos while steady state models may not be able to show the same. Such studies reviewed by Toomre [14] would be of relevance in this context. (see also Lin and Bertin [14] and Ref. 4, Vol. TI).

The existence of intrinsic chaos and quasilinear diffusion can accelerate the process of relaxation of a galaxy to a ilaxwellian distribution. Emission and absorption of density waves (see in the plasma context eg. Krall and Trivelpiece [15]) by star points with random and quasilinear diffusion motions would take place and a steady state balancing of these two processes would be very important in determining the nature and limits of relaxation of a galaxy. The waves because of their collective nature induce chaos; chaotic and overall motion of stars contributes to the spectrum of waves emitted into the star collection. A dynamic equilibrium where chaotic motions play a crucial role in steady state relaxed behaviour is then very important.

The model presented by Eq. (4) here is that of 1 inear transport equation. This is a very specialised form of the Master equation for irreversible dynamics. If such a Master equation could properly be set up, a more general criticality study in the onset of chaos can be described as in Ref. 16. Finally, it should be mentioned that $\mathrm{Eq}$. (4) or its solutions can also be generalised if more complicated star clusters be found in phase space; the basic diagram methods to determine propagators as in Ref. (17) should be kept in mind.

\section{REFERENCES}

[1] J.Binney and S.Tremaine (Princeton University Press, Princeton, 1987).

[2] James Binney, 'Stellar Dynamics in Evolution of Galaxies Astronomical Observations p.95 (Springer, Berlin, $1988)$.

[3] William Saslaw, 'Gravitational Physics of Stellar and Galactic Systems'. (Cambridge University Press, Cambridge, 1985 ).

[4] A.M. Fridman and V.L. Polyachenko, 'Physics of Gravitating Systems', Vols. I\& I I (Springer, New York, 1984).

[5] A.J.Lichtenberg and M.A. Lieberman "Regular and Stochastic Motion", (Springer, New York, 1983).

[6] J.Guckenheimer and Philip Holmes "Nonlinear Oscillattions, Dynamical Systems and Bifurcations of Vector Fields" (Springer, New York, 1983).

[7] Ta-You Wu "Kinetic Equations of Gases and P1asmas" (Addison Wesley, Reading, 1966). Section 5.8,p.138.

[8] K.M.Case and P.F. Zwifel "Linear Transport Theory" (Addison Wesley, Reading, 1967). 
[9] K.M. Case,Phys. F1uids, 21, 2499 (1978).

[10] T.Y. Petrosky, Phys.Rev. A32, 3716 (1985).

[11] R.Uma and D.Subbarao, Phys.F1uids, 82,1154(1990).

[12] R.L. Dewar, J.Phys. Á9, 2043 (1976).

[13] R. Uma and D. Subbarao, Bul1. Am. Phys. Soc. 3ㄷ, Nov.1990 (In Press).

[14] A. Toomre, Ann. Rev. Astron.Astrophysics. 15,437-478 (1977) (see particularly the work of Hunter (1969) referred here); also C.C. Lin and G.Bertin "Galatic Dynamics and Gravitational Plasmas" in Advances Applied Mechanics 24, Ed. John W. Hutchinson and Theodore, Y. Wu (Ac ad emic, Orlando, 1984 ).

[15] N.A. Krali and H.W. Trivelpiece, "Principles of Plasma Physics" (McGraw Hill, New York,1966) (last chapter).

[16] I. Dana, N.W. Murray and I.C. Percival, Phys.Rev.Lett. $62,233(1989)$.

[17] R.Balescu, "Statistical Mechanics of Charged Particles" (Interscience, New York, (1963)). 